\title{
New clot busters threaten Genentech's tPA
}

Genentech's (S. San Francisco, CA) tissue plasminogen activator (tPA), alteplase, appears to have survived another challenge to its dominance of the $\$ 355$ million thrombolytic drug market. The results of the 15,060patient GUSTO III trial (third global use of strategies to open occluded coronary arteries trial) reported at the 46th annual meeting of the American College of Cardiology (ACC, Anaheim, CA) showed that heart attack patients have virtually the same chance of survival whether they are treated with the Genentech drug or reteplase (RPA), produced by Boehringer Mannheim (Mannheim, Germany): 30 days after an initial heart attack, 7.43\% of the reteplase patients and $7.22 \%$ of the alteplase patients had died, a statistically insignificant difference. The $\vec{a}$ study investigators could not agree on whether the two drugs are equivalent.

For Genentech, the result provides satisfying evidence that alteplase should remain the gold-standard for treatment of heart attack patients, "The trial failed," notes Paul Laland, spokesperson for Genentech. "It set out to prove that RPA saved more lives than IPA, but failed to prove the hypothesis." "The trial is not a reason for doctors to begin prescribing anything else," he adds. But, unsurprisingly, Boehringer Mannheim staff interpret the trial differently. "Basically, the trial showed that both drugs are the same in terms of efficacy and side effects" says Stephan Fischer, director of biotechnology R\&D at Boehringer Mannheim. And that, says Fischer, will mean that reteplase's ease of administration will make it the drug of choice.

Reteplase is given as two bolus injections 30 minutes apart, whereas alteplase must be administered as a 90-minute infusion with weight-adjusted dosing. "When treating [heart attack] patients, the complications of infusions and calculations of the different speed of infusion can lead to problems with compliance [by patients]. The double bolus without weight adjustment is well accepted and is a major advantage of [reteplase]," notes Fischer.

Reteplase contains only two of the five protein domains present in natural tPA (and alteplase), the kringle-2 and protease domains; the F, E, and kringle-1 domains have been removed. In previous trials, reteplase was better than alteplase at restoring

Sylvia Davidson is a freelance writer working in London.

\section{IMAGE \\ UNAVAILABLE \\ FOR COPYRIGHT REASONS}

tPA is the leading therapeutic for dissolving clots in blood vessels (inset) in myocardial infarction or massive pulmonary embolism.

complete blood flow. The alterations have increased the half-life of the protein in human plasma from 3-6 minutes to $16-18$ minutes, thereby allowing the bolus administration method. Experiments have also shown that the absence of fibrin-binding domain (the $\mathrm{F}$ domain) makes reteplase more effective in disrupting clots, according to Fischer. "The tPA sticks on the surface of the clot because of fibrin binding, whereas reteplase is washed into the clot and can activate plasminogen not only on the surface, but also within the clot."

That same property might, however, have caused serious unwanted bleeding as a side effect. However, the GUSTO III results indicated that the side effects of the two drugs are in fact comparable; according to Fischer,

Table 1. Variants of tPA under development.

Drug
$\begin{aligned} & \text { Monteplase (second- } \\ & \text { generation tPA) }\end{aligned}$

having equivalent side effects is "not disappointing."

The importance of ease of use-even for emergent drugs like tPA-is highlighted by the development of several other tPA variants (see Table 1). Genentech's second-generation tPA, TNK-tPA, can be administered as a single injection. Bristol-Myers Squibb (BMS; New York) is about to begin phase III studies with NPA. Originally developed by Genetics Institute (Framingham, MA), NPA is a variant of $\mathrm{tPA}$ that is missing the $\mathrm{F}$ and $\mathrm{E}$ domains and has amino acid changes at three sites. Citing a phase II study presented at the ACC meeting, Paul Chew, medical director at BMS, says that NPA can be administered as a single injection, something that is "very important" from a practical point of view. Boehringer Mannheim is continuing to investigate the structure of plasminogen activator and to develop further variants. Another variant of tPA is the nitrosylated tPA, developed by Nitromed (Boston, MA), which, according to Gordon Letts, Nitromed's vice president of $R \& D$, combines the fibrinolytic activity of tPA with the antiplatelet effect of nitric oxide.

The consistent $7-7.5 \%$ mortality rate found with both alteplase and reteplase in the GUSTO III trial may indicate that the combination of thrombolytic agent and heparin used may have reached a ceiling of effectiveness. "It is now necessary to optimize the drug regimen, to move away from heparin alone to the use of platelet aggregation inhibitors," says Fischer. However, Eric J. Topol, chairman of the Cleveland Clinic and chairman of the GUSTO III trial, saw a more pragmatic route to the same end: He noted that if heart attack patients were treated one hour earlier than at present, mortality could be reduced by $15 \%$, a figure that a new therapy would find very difficult to beat.

Sylvia Davidson 